\title{
Psychosocial aetiology of chronic disease: a pragmatic approach to the assessment of lifetime affective morbidity in an EPIC component study
}

\author{
P G Surtees, N W J Wainwright, C Brayne
}

\begin{abstract}
Objectives-The Health and Life Experiences Questionnaire (HLEQ) was developed for use in a prospective cohort study of 25000 men and women living in Norfolk and forms a component study of the European Prospective Investigation into Cancer and Nutrition (EPIC). The HLEQ includes an assessment of mood status over the life course allowing a limited capacity for the imposition of diagnostic criteria to enable eventual evaluation of mental health status for chronic disease outcomes. This paper reports estimates of HLEQ Major Depressive Disorder (MDD) prevalence and compares them with those obtained through interviewerbased methods. In addition evidence for the impact of recall, clustering or cohort effects on these estimates are examined. Participants-3491 eligible respondents to EPIC in Norfolk, aged 45-74 years, recruited from the first five general practices who completed the HLEQ.

Main results-MDD prevalence estimates were found to be closely comparable to those obtained recently (by interview) in the UK and to those lifetime MDD rates determined through international studies. Risk of MDD onset was found to vary with age as expected from earlier studies using interviewer-based assessments. Limited evidence was found to show that the distribution of first onset MDD episodes were compressed during the immediate pre-assessment period. Results were also consistent with previous evidence demonstrating the raised risk of MDD among women and of the decline in gender differences with advancing age.

Conclusions-These results suggest that estimates of putative MDD diagnostic status, derived through the HLEQ, and of associated demographic risk are similar to those derived by more intensive and costly assessment methods. Implications for the future study of MDD both as an outcome and as a risk factor for chronic disease are discussed.

(F Epidemiol Community Health 2000;54:114-122)
\end{abstract}

The past two decades have seen the development of formal diagnostic criteria for the classification of psychiatric conditions with their representation in interview assessments designed to be applied in a wide range of settings. ${ }^{1-6}$ This evolutionary process has been driven, at least in part, by the need to achieve gains in diagnostic precision, to facilitate national and international comparisons of the prevalence of disorder, to enable study of the aetiology of psychiatric conditions and to provide as firm a foundation as possible for the provision of psychiatric services. In addition, the continued absence of any reliable biological tests to detect psychiatric disorder, has provided further impetus to refine diagnostic criteria and allied measurement procedures. Estimates of the prevalence of psychiatric conditions derived through the application of identical criteria and assessment approaches are now available from many centres. Such studies have revealed the consistency across studies with which specific factors confer heightened risk for some conditions (for example, gender for major depressive disorder), and at times the extreme (and largely unexplained) variability in prevalence estimates identified through international collaborative studies. ${ }^{78}$

In the wake of this work, potential weaknesses in the diagnostic assessment process have been raised. ${ }^{9-13}$ These include the design of appropriate procedures to test the reliability and validity of lay administered diagnostic instruments and the capacity of a single retrospective interview to provide a reliable and valid estimate of psychiatric morbidity over a lifetime. Underscoring these weaknesses have been the results of test-retest designs that have yielded (for example) relatively poor levels of agreement between (previous month) lay and clinical assessments. ${ }^{9}$ While the objective of this work has been "...the desire to have an instrument that will, as closely as possible, replicate a psychiatrist's diagnoses for situations where the use of psychiatrists is impracticable or impossible" (page 666), ${ }^{14}$ such objectives are constrained through the likelihood that the clinician's interview is an "erratic standard", that test-retest designs need to consider both clinical change over the test interval (and control for order effects), and consider the statistical difficulties imposed through the base-rate problem. ${ }^{15}{ }^{16}$ Work cited by Robins ${ }^{15}$ suggested that a structured questionnaire, used as part of the neuropsychiatric screening of prospective inductees (throughout the United States during the final year of the second world war), "produced much more uniform results across centers than did the physicians charged with using their clinical expertise to predict which young men were poor psychiatric risks for success in the armed services." (page 920). Subsequent work concerning depressive disorders in 
particular, has shown inter-rater reliability to vary according to the number and severity of episodes; with estimates of the reliability of a recurrent mild depressive disorder and reliability over a one year period in a non-clinical population to be rather low ( $\kappa$ values 0.37 and 0.34 respectively). ${ }^{17}{ }^{18}$ The definite clustering of (for example) episodes of major depressive disorder (MDD) during the years immediately before times of assessment has also caused concern. Cumulative lifetime rates of MDD by birth cohort from several large epidemiological studies, using formal diagnostic criteria allied to structured interview approaches, have shown the distribution of episodes of disorder compressed within the very few years just before assessment, rather than distributed as expected over extended time periods. ${ }^{13}$ While epidemics of affective disorder could explain these findings, this seems unrealistic. These overall results challenge psychiatric epidemiologists to either abandon attempts to obtain a trustworthy account of a psychiatric history through a single retrospective assessment or to identify ways of improving assessment approaches; an issue that has particular salience for setting the framework within which the relation between psychiatric and physical disorders can be assessed. ${ }^{19}$

In parallel with these developments in psychiatric epidemiology, prospective cohort studies designed to provide clues to the origin of chronic disease have begun to include social and psychological measures to be considered alone and in combination with those more typically representative of biological, nutritional or genetic risk. The ideas that have contributed to this work stem partly from recent demonstrations that immune function interacts with behavioural, neural and endocrine processes; speculation then suggests that these interactions may underpin the evident role of social and psychological factors in the onset of (for example) infectious autoimmune and neoplastic disease. ${ }^{20-22}$ Results from some of these studies and the conclusions from literature reviews have suggested the potential significance of the psychosocial factors for incidence, for example of cardiovascular endpoints, ${ }^{23-29}$ and possibly for cancer although the evidence here is more equivocal. ${ }^{30-35}$ One feature of this work has been the growing suspicion that a history of affective disturbance may have implications for improving understanding of chronic disease aetiology and possibly of mortality. ${ }^{36-43}$ However, this suspicion is borne of results typically dependent upon measures of affective phenomena that bear little or no resemblance to those diagnostic indicators of affective disorder now commonly used in psychiatric epidemiology. Recent evidence using stringent scoring of the presence of depressed mood, ${ }^{44}$ has provided more compelling evidence that a chronic history of depressed mood (at least in older persons) increases cancer risk. To attempt to clarify the nature of the relation between affective health status and chronic disease therefore presents a number of special challenges. In particular, assessments of the very large populations typically required for incidence studies of chronic disease ensure that the approaches developed within psychiatric epidemiology are inappropriate given the specialist skills needed and their demands on participant time over and above measurement of the core areas of interest. One approach to bridge the gap may be through the development of respondent self assessment methods designed to be as closely tuned as possible to current diagnostic criteria for specific psychiatric conditions. This paper aims to describe such an approach to the self assessment of depressive disorder in the context of a component study of the European Prospective Investigation into Cancer and Nutrition (EPIC). ${ }^{45} \mathrm{~A}$ further aim is to determine the extent to which some of the concerns raised by others in the context of interviewer-based assessments of depressive disorder in epidemiological cohorts are also found to be relevant in the EPIC in Norfolk cohort.

\section{Methods}

DESIGN-EPIC IN NORFOLK

EPIC is an ongoing multicentre prospective cohort study investigating the importance of nutritional and other factors on chronic disease development in nine European countries with populations distinguished by variations in dietary habits and cancer risk. The EPIC in Norfolk study, administered from Cambridge, is designed to measure the incidence of chronic disease (including cancer, cardiovascular disease, diabetes, rheumatoid arthritis and osteoporosis), in a population sample of around 25000 people aged 45-74 years at recruitment living in Norfolk, an area geographically centred on Norwich in East Anglia. The sample has been recruited through general practitioners with core data collected on diet, haematological indices, physical activity, reproductive history, medical and family history and lifestyle through a variety of questionnaires and other procedures. ${ }^{46-48}$ The relative absence of measures of psychosocial factors in the study was addressed through the development of the Health and Life Experiences Questionnaire (HLEQ). This was designed to permit the exploration of a wider range of causal models relating human behaviour to chronic disease outcomes than would otherwise have been possible.

THE HEALTH AND LIFE EXPERIENCES

QUESTIONNAIRE (HLEQ)

The HLEQ was designed after an extensive review of the literature reporting relations between broadly defined psychosocial factors and chronic disease (principally cancer and cardiovascular disease) and according to the principles of questionnaire design advocated by Dillman. ${ }^{49}$ During 1995, after pre-piloting, two pilot studies were undertaken with each based upon random samples of 50 patients of similar age to those entering the EPIC in Norfolk study. These samples were drawn from patient lists of two group general practices serving East Anglian communities not participating in the EPIC study. The pilot studies were designed to test procedures, to assess the willingness of 
respondents to complete the questionnaires, to detect ambiguities and to overcome misinterpretations of items through subsequent redesign. After a reminder letter, $78 \%$ of the pilot samples returned completed questionnaires. Pilot testing resulted in the production of a 36 page A4 format questionnaire divided into seven assessment areas, namely; health and daily activities, work, social life, mood, lifetime events, childhood experiences and personal beliefs. These areas included the 36-item Short-Form health survey questionnaire (SF36), developed from the Rand Corporation's Health Insurance Experiment (HIE) undertaken in the United States, ${ }^{50}$ measures of social support as used in the Whitehall II study ${ }^{51}$ and of relationships in childhood, adapted from those used in the Midlife Development Inventory (MIDI), developed by the MacArthur Foundation Research Network on Successful Midlife Development for a national survey of Midlife in the United States (MIDUS). These measures were included to permit future contrasts in findings with those from EPIC. The HLEQ is sent to all EPIC respondents some 18 months to two years after their recruitment to the study and their completion of an initial EPIC questionnaire, regardless of their subsequent study compliance. This paper presents results from the mood section of the HLEQ by a sample of EPIC participants from the first five general practitioner group practices.

THE MEASUREMENT OF MOOD STATUS OVER THE LIFE COURSE

The HLEQ includes a restricted assessment of the mood status of a respondent over their lifetime. This is assessed through a structured self assessment approach to psychiatric symptoms embodying rules from the fourth edition of the Diagnostic and Statistical Manual of Mental Disorders (DSM-IV), ${ }^{6}$ but limited only to MDD and generalised anxiety disorder (GAD). Only results concerning MDD will be presented here. The modular assessment method was informed through the design of short-form symptom scales derived from the National Comorbidity Survey (NCS), ${ }^{52}$ through discussion and subsequent personal communication with the principal investigator to the NCS and modified after subsequent pilot studies.

The HLEQ mood section was designed to identify those thought likely to meet putative (anxiety or depressive) DSM-IV diagnoses at any time in their lives and to provide evidence of chronicity. As it was beyond the scope of the questionnaire to check fully for DSM-IV specified exclusions, only criteria "A" (symptoms) and " $C$ " (clinical significant distress or impairment) were assessed for MDD episodes. The assessment procedure depends upon respondents initially disclosing details of their most recently experienced episode of depression (if more than one) that has lasted for two weeks or more and fulfils the required concurrent symptom criteria. Additionally, operational criteria for clinically significant impairment and help seeking was included in the self assessment and respondents were asked to estimate episode onset and (if appropriate) offset timings. Where the first episode was reported, further questions followed that were designed to provide estimates of the age of first onset and of outline details of any interval episodes that may have been experienced between the first and most recently reported, with respondents permitted to report $1,2,3,4$, at least "..." or "too many episodes to remember". The complete sequence of questions is shown in the appendix. The questioning process is dependent upon the respondent acquiring a "map" of their most recent affective episode, this then providing the cognitive template to carry back in time to aid their recall of any previous episodes.

Given the views expressed concerning morbidity estimates derived from interviewerbased assessment approaches, ${ }^{13}$ it is very likely that those derived through self report assessment, no matter how carefully designed, are likely to share similar problems, and probably to greater degree. This of course would have implications for evaluating their contribution for understanding chronic disease outcome. This paper therefore sets out to establish firstly, whether estimates of MDD prevalence are congruent with or vary from those obtained through interviewer based methods (and of how that differs by gender), and secondly what evidence there may be for problems of episode recall, clustering or cohort effects in the HLEQ interim data.

\section{ANALYSIS}

Kaplan-Meier survival estimates gave a measure of lifetime prevalence, plotted as percentage with disorder by study time (age), and a person years approach was used to estimate the annual incident episode rate by age. For the latter, age was divided into eight 10 year bands, with each individual contributing 10 person years to each age band up to their current age. Reported episodes were dated according to age of first onset and age at last episode and the total number of episodes divided over the intervals during which these episodes were experienced. The incident episode rate in each age band was then taken as the total number of episodes experienced divided by the total person years in that age band. An estimate of annual prevalence was subsequently derived by assuming uniform episode duration by age so that prevalence was equal to incidence multiplied by a constant (to represent average episode duration over the whole sample). A direct and reasonable estimate of episode duration was not available from these data, so an indirect approach was taken by equating annual prevalence in the age range 48-79 estimated from the curve (taking age distribution into account) and the observed current (12 month) EPIC-HLEQ prevalence estimate (for the age range 48-79). The ratio of lifetime to annual prevalence was then explored graphically to reveal how it might be expected to vary by the age ranges of different studies. Gender differences were determined as odds ratios with $95 \%$ confidence intervals (CI). Missing data 
resulted in the exclusion of 76 observations where putative MDD diagnoses could not be determined and a further 29 where age at first onset was not reported. In calculations of annual prevalence, total numbers of episodes were truncated to a maximum of five (both to avoid complications in dealing with the response "too many to remember" and to limit the influence of the few people who report (perhaps unrealistically) large numbers of episodes over short time periods). Further loss of data was avoided by substituting the mean number of episodes (conditional on having experienced at least one episode) for those where diagnosis of MDD was achieved but the total number of episodes was unknown ( $\mathrm{n}=$ 23 ); where age of first onset was unknown ( $\mathrm{n}=$ 29) these observations were recovered and episodes were divided from age band 1 to age at last episode and similarly, where age at last episode was unknown $(n=71)$ this was taken as age at questionnaire completion.

\section{Results}

Analyses were based upon HLEQ returns from respondents contacted through the first five general practices working with the EPIC study in Norfolk. A total of 11847 people, registered at these practices were invited to participate in the EPIC project of which $43 \%$ consented to their involvement. This group (with further exclusion of those who had subsequently died, moved out of the area or indicated refusal to any further approach by EPIC), were mailed the HLEQ ( $\mathrm{n}=4555)$. Analyses were based upon an available sample of 3491 people aged between 48 and 79 years at the time of questionnaire completion, representing a response rate of $77 \%$, albeit around $30 \%$ of the original (eligible) general practice population. A comparison of HLEQ respondents with those in the original general practice population who did not complete the HLEQ for whatever reason $(\mathrm{n}=8356)$, showed that HLEQ respondents included a greater proportion of women $\left(56 \% v 50 \%, \chi^{2}=38.3\right.$ on 1 df, $\mathrm{p}<0.001)$ but that no differences were found in terms of mean age (both 62.3). Other work $^{48}$ has found that the EPIC in Norfolk cohort is representative of the population studied in the Health Survey of England, ${ }^{53}$ in terms of anthropometric factors, blood pressure and serum lipids but has fewer smokers.

An indication of possible bias in terms of depression status was available from comparisons with (contemporaneous) scores on the Mental Health (MH) scale of the SF-36, ${ }^{50}$ a general population measure of perceived health. Two studies seeking to establish UK population norms for the SF-36, one in Sheffield $^{54}$ and the other centred around Oxfordshire ${ }^{55}$ provide data for age matched comparisons, restricted across common age ranges. Mean (age matched) SF-36 MH scale scores were 77.6 for EPIC-HLEQ respondents as compared with 73.1 in Sheffield (common age range 45-74) and age-gender matched comparison between the HLEQ and Oxfordshire samples (common age range 45-64) gave mean scores of 76.3 and 75.2 , respectively (with scores of 77.9 and 77.1 for men and 75.2 and 73.8 women). It has been argued that SF-36 norms differ across geographical regions,${ }^{56}$ so further comparison was made with pooled data from the HLEQ pilot study samples (likely to be representative of SF-36 norms in the local EPIC area). This contrast revealed a mean (SD) SF-36 MH score of 77.6 (16.2) in the HLEQ interim sample and 73.4 (19.6) from the pilot study samples (based on 71 completed SF-36 scores from a total of 100 questionnaires). These SF-36 MH scores also provided a means of validating the HLEQ based MDD diagnosis and episode timing relative to assessment. Mean SF-36 scores were found to vary considerably by putative MDD diagnosis, resulting in a mean $\mathrm{MH}$ score of 78.9 (15.1) in those respondents with no lifetime depression; 77.3 (15.0) in those with a lifetime diagnosis but where no episode was experienced in the year before HLEQ completion; 62.8 (18.6) in those where the depressive episode had been experienced in the previous year but was not current and 44.5 (19.3) in those satisfying current putative DSM-IV MDD criteria. This latter result corresponds closely with what has been found in relation to SF-36 MH scores in those with clinical depression in the Medical Outcomes Study population (mean 46.3)..$^{50}$

Taken together these results reveal that the EPIC-HLEQ interim sample includes a higher proportion of women but has similar age distribution to the base population, is healthier in terms of scores on the $\mathrm{MH}$ scale of the SF-36, but these differences were not large when compared with variations in SF-36 MH scores across HLEQ MDD diagnostic groups.

\section{MDD PREVALENCE}

Putative DSM-IV criteria gave estimates of $2.3 \%$ for current (2 week) MDD prevalence, $4.2 \%$ for the 12 months preceding questionnaire completion and $13.9 \%$ over the lifetime. Publicly available data from the OPCS (now ONS) surveys of psychiatric morbidity in Great Britain $^{57}$ provided a basis for comparison of current (2 week) rates of MDD. Prevalence estimates, restricted to the common age groups across the OPCS and EPIC-HLEQ studies (age range $48-64$ ) were $2.2 \%$ for the OPCS survey as compared with $2.9 \%$ for the EPICHLEQ. Figure 1 shows a comparison of MDD prevalence estimates, age standardised to the United States five site Epidemiologic Catchment Area (ECA) study, obtained in a group of international psychiatric epidemiological studies undertaken during the $1980 \mathrm{~s}^{8}{ }^{8}$ Rates were determined according to diagnostic criteria closely approximating those used here, but based upon interview gathered data. While it was not possible to age standardise the EPICHLEQ rates to the younger samples shown here, the very wide variation in the interviewerbased age standardised MDD rates can be seen as placing the HLEQ based rates within the bounds of what might reasonably have been expected. More recently this variation in interviewer rates has been extended with publication of the results of the NCS survey (DSM- 
Figure 1 Comparison of MDD prevalence rates between a group of international studies based upon a common interviewer-based method (rates shown with $95 \%$ CI). (A) Current (12 month) prevalence and $(B)$ lifetime prevalence. (Study abbreviations are as follows: Tw: Taiwan; $K$ Korea; PR: Puerto Rico; US-ECA: United States, ECA; F: France; WG: West Germany; C: Canada; NZ: New Zealand; I: Italy; L: Lebanon. See Weissman et $a l^{8}$ for further details).

Figure 2 Lifetime and annual prevalence rates of $M D D$ and lifetime:annual prevalence rate ratio by age.

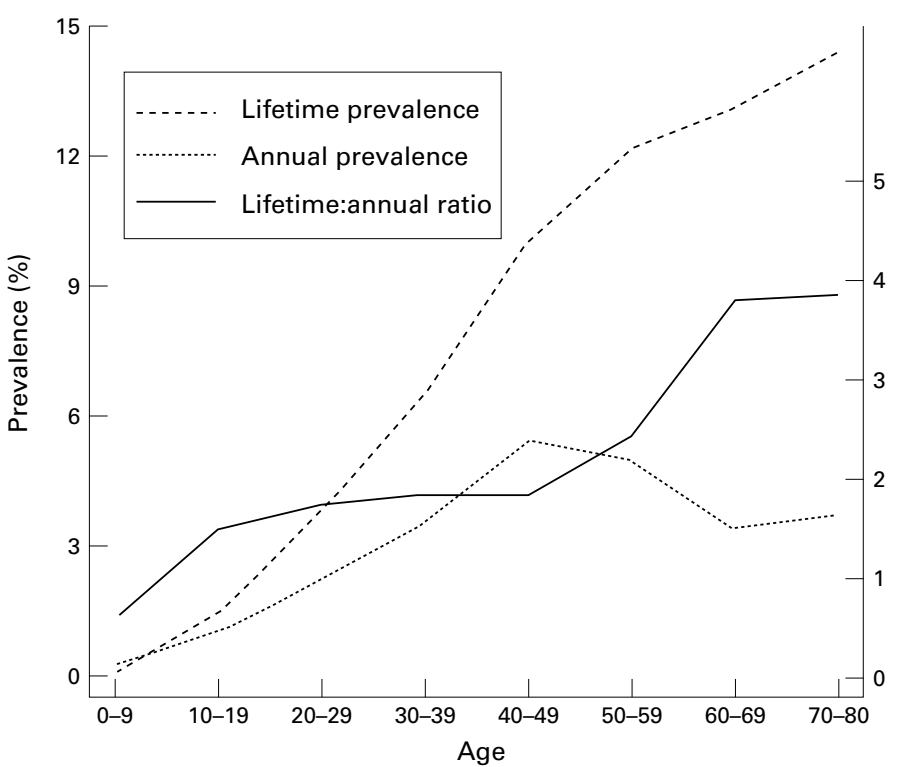

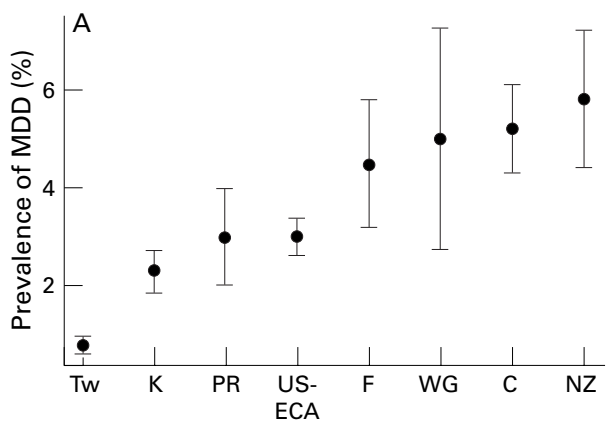

IIIR 12 month MDD rate $10.3 \%$; lifetime MDD rate $17.1 \%) .^{52}$

During the 1980 s, attention was focused upon the magnitude of the ratio of current to lifetime prevalence estimates of psychiatric disorder derived from studies using structured interview approaches allied to diagnostic criteria. $^{10}$ This raised serious questions as to the accuracy of the estimates of lifetime morbidity as results suggested that the likelihood of meeting psychiatric disorder criteria during a period of six months to a year pre-interview, was about half the lifetime likelihood of experiencing a psychiatric disorder. This result was considered perhaps unlikely given the implications for recurrence/ chronicity that would need to be satisfied for such conditions. While others have raised issues concerning aspects of the interview techniques used in the 1980 s to recall episodes of disorder over extended time periods, and have modified assessment techniques with consequent impact upon prevalence estimates, rate ratios derived from such later work have remained within the bounds of the earlier concerns. Given the relative consistency, but remaining controversy over such findings, these issues were considered in these EPIC - HLEQ data.

Overall, these data suggest a rate ratio of 3.3:1 indicating that the likelihood of meeting MDD in the previous 12 months was about $30 \%$ of the lifetime MDD likelihood. Both the

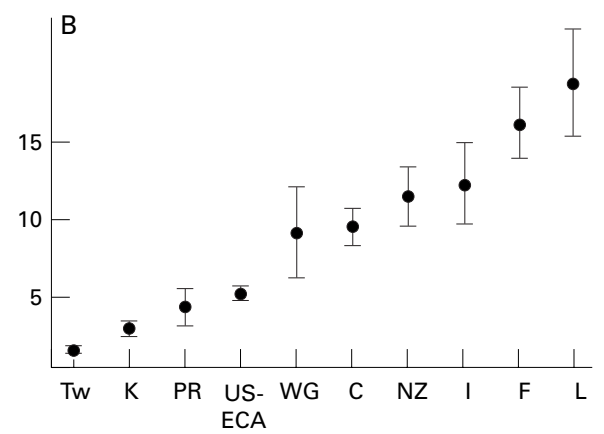

Epidemiological Catchment Area study $(\mathrm{ECA})^{58}$ and the younger cohort assessed in the more recent NCS study (where modifications were made to improve respondent recall procedures), yielded results suggesting that almost 6 of every 10 of those respondents that had ever met MDD criteria had done so in the 12 months immediately before assessment. To investigate how the age ranges of different epidemiological studies might influence the rate ratio, the cumulative lifetime and annual MDD prevalence rates by age were calculated and are shown along with their ratio in figure 2 . The results show that MDD prevalence in the EPIC-HLEQ sample reaches a peak at age 40-49, declining thereafter. Correspondingly, the rate of increase in lifetime prevalence reduces after age 50 . Hence the ratio of lifetime to annual prevalence appears lowest for the youngest age groups and remains relatively stable up to age 50 as both lifetime and annual prevalence increase. Thereafter, as annual prevalence declines while lifetime prevalence continues to increase, there is a sharp increase in the rate ratio. This result implies that a larger rate-ratio is to be expected in more elderly samples as in those completing the EPICHLEQ and lends some support for the apparently unlikely ratios of around 2:1 in the studies of younger cohorts.

MDD FIRST ONSET: RECALL, EPISODE CLUSTERING AND COHORT EFFECTS

Figure 3 shows evidence of the capacity of the HLEQ to document first episodes of MDD. The figure displays the number of MDD first episodes reported in each of three birth cohorts (those aged 48 to 55 at the time of self assessment, those 56 to 64 and those over 65 years), and according to the elapsed time since first onset. A total of $444 \mathrm{MDD}$ first episodes were dated and extended over a retrospective period exceeding 50 years. For all cohorts there was a trend for a greater number of first episodes to be reported during those decades closest to assessment. This trend was most pronounced in the youngest cohort reflecting either compression attributable to recall bias or the natural age distribution of MDD onsets (the younger cohort having more recently experienced a period of heightened risk) and barely evident in the middle cohort. More importantly, these data show that first onset MDD episodes were distributed over a very long time period, in marked contrast with evidence for episode clustering during the few years just 
Figure 3 Total number of reported first onsets of MDD by time since episode occurrence and by birth cohort.

Figure 4 Cumulative incidence of first onsets of $M D D$ by birth cohort for (A) US-ECA and (B) EPIC-HLEQ, interim cohort.

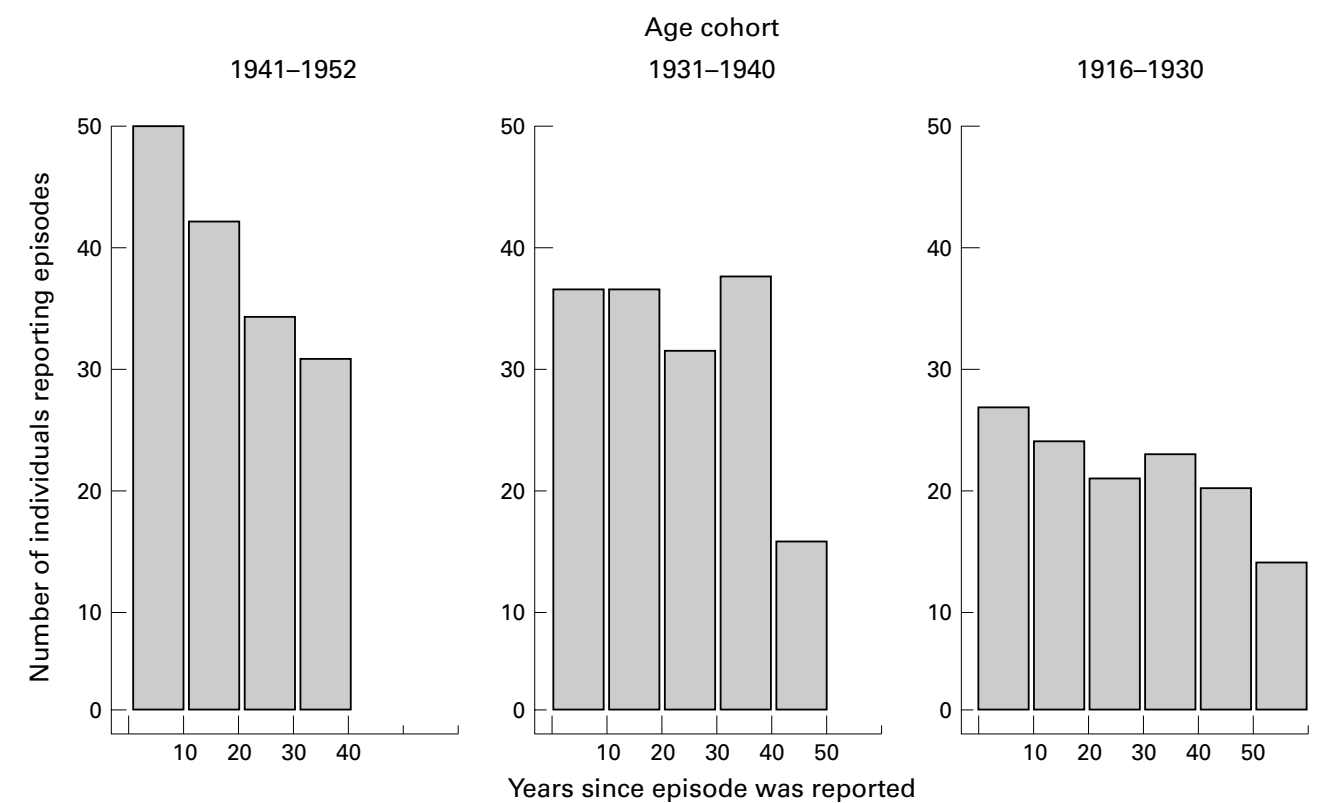

before interview for all birth cohorts in some other studies ${ }^{13}$ and provide some reassurance concerning the distribution of first onset episodes detected over time.

The change in cumulative risk of MDD first onset by birth cohort is shown in figure $4 \mathrm{~B}$ for the EPIC cohort and provides a further comparison with the US-ECA study. ECA data $^{7}$ are reproduced in figure $4 \mathrm{~A}$ for birth cohorts overlapping with the EPIC cohort and the cumulative risks by age by birth cohort for these two studies are shown side by side. While EPIC-HLEQ based rates were consistently higher than corresponding ECA age period cohort rates, the temporal pattern and tendency for younger cohorts to report higher rates remained the same. Given that the EPICHLEQ assesses the equivalent age period effects some 15 years after the ECA assessments were made, this provides further reassurance of its capacity to document first episode onsets over the lifetime.

A

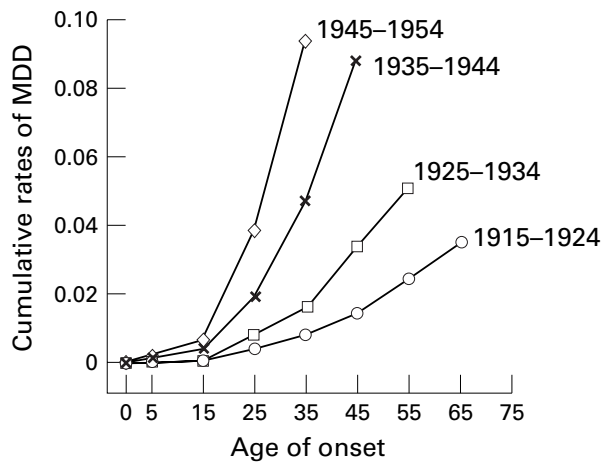

GENDER RISK

Perhaps the most consistent finding from the study of depressive disorder has been the demonstration of the excess risk among women; typically twofold higher than in men. ${ }^{58}{ }^{59}$ However, convincing explanation for this finding has not been achieved despite research strategies that have adopted social, psychological, biological and increasingly genetically sensitive approaches. It has been argued that on the balance of current evidence, insight into this differential risk may be gained only through the adoption of a developmental perspective that takes special account of the interaction between changing social experience over time and individual psychological (particularly cognitive) attributes. ${ }^{6061} \mathrm{~A}$ primary task therefore was to establish firstly, whether the EPIC-HLEQ data revealed results that were consistent with those from other interviewer-based studies in confirming the excess risk of MDD in women over men, and further whether the excess (if found) was

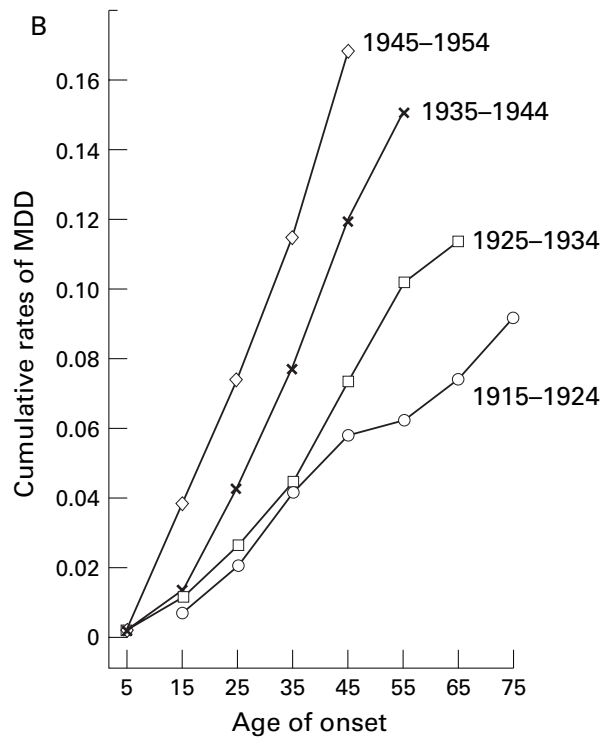




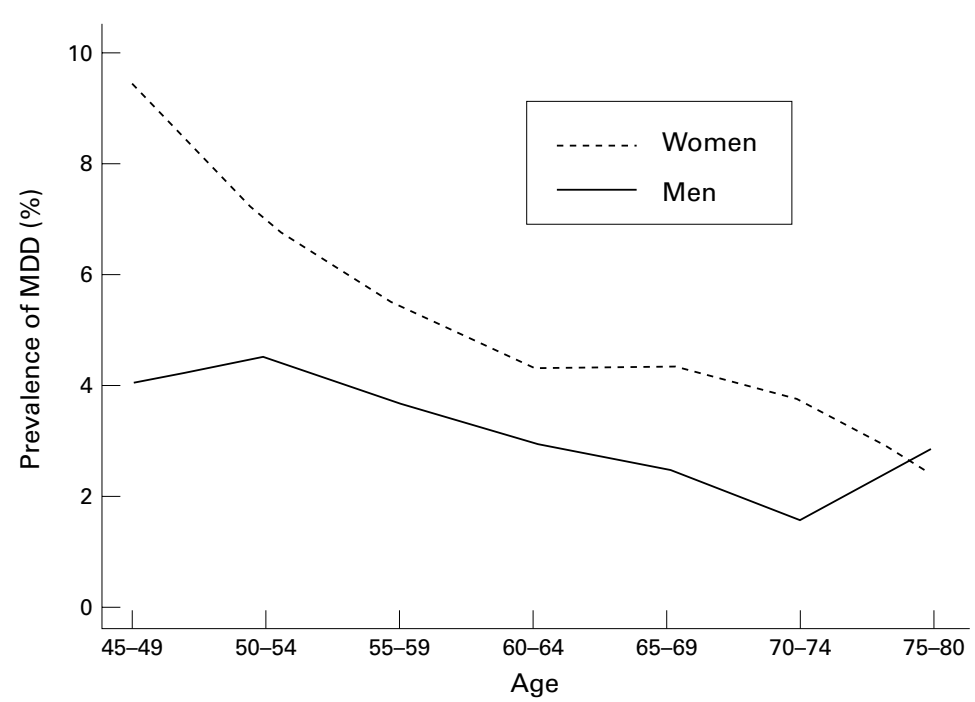

Figure 5 Current (12 month) $M D D$ prevalence by age and gender.

most pronounced during the childbearing years; secondly whether there was any evidence for the more recently observed suggestion that gender risk declines (and reverses) in post-menopausal women. ${ }^{62}$ Analysis revealed that the female lifetime and 12 month MDD rates were $16.6 \%$ and $5.1 \%$ respectively and for men, $10.4 \%$ and $3.1 \%$ resulting in a female:male odds ratio of 1.7 (95\% CI 1.4, 2.1) and $1.7(1.2,2.5)$ for lifetime and 12 months prevalence, respectively; results largely coincident with those obtained in the recent NCS study. ${ }^{52}$ Figure 5 displays the MDD current (12 month) prevalence rates by gender. This shows a slight decline in MDD prevalence with increasing age for men with a much more pronounced fall in the female rate. This corresponds closely to evidence recently gathered internationally. ${ }^{7}$

\section{Discussion}

A recent World Health Organisation ${ }^{63}$ annual report has suggested that by the year 2005 in European Union countries alone there may be a $33 \%$ increase in lung cancers in women and a $40 \%$ increase in prostate cancers in men. Accumulating, but still inconclusive evidence, on the relation between diet and cancer and on the role of physical activity, smoking and obesity on health has resulted in the WHO calling for an intensified and sustained global campaign to encourage healthy lifestyles and to seek ways of modifying what are seen as the main risk factors.

EPIC in Norfolk is a large scale prospective cohort study designed with the primary objective of extending understanding of the association between diet and cancer in middle and later life. That objective is being progressively broadened to embrace other chronic disease end points and to include new measurement areas such as the baseline assessment of psychosocial factors through the HLEQ. The impetus for extending measurement to these areas has come from a growing literature suggestive of the role of social and psychological factors for chronic disease outcomes. However, reported findings from much of this work remain controversial because of measurement and study design weaknesses that pervade the
KEY POINTS

- Intriguing and increasingly persuasive evidence has been mounting for depression as a predisposing factor for cancer and cardiovascular disease.

- Self assessment of mood status is reported in a component study to the European Prospective Investigation into Cancer and Nutrition (EPIC).

- Prevalence and risk factor estimation is found to be comparable with expectations derived from interviewer-based international psychiatric epidemiology projects.

- Future evaluation of the role of psychosocial factors in chronic disease incidence should be aided by the procedure reported.

area, discussed in detail by Fox. ${ }^{30}{ }^{64}$ Recent literature reviews have concluded, for example, that there is little support for a link between depression and cancer incidence, ${ }^{34}$ but that the area is bedevilled by methodological weaknesses. This work is based (almost) exclusively upon follow up studies that have used snapshot aggregate symptom assessments of mood state, typically focused only upon very brief periods before assessment. ${ }^{35}$ The significance of any findings from this work remains uncertain given their foundation upon measures that may reflect only brief and transient states and in consequence be relatively poor indicators of their true (underlying) risk status. The recent work focusing upon chronic depressed mood and subsequent cancer risk in older persons, ${ }^{44}$ serves to highlight the need for greater attention to measures of psychological health. This methodological limitation also underpins the association found between depressed mood and heart disease, ${ }^{23} 38$ though a recent report based upon the use of clinical depression criteria in a prospective design have supported the earlier evidence for increased risk of myocardial infarction in those with affective state histories. ${ }^{65}$ To begin to clarify the uncertain relation between affective health and chronic disease incidence requires refinement of measures of complex risk factors to reduce uncertainty associated with findings.

This paper has focused on the use of a questionnaire approach to the assessment of affective state in the context of a large scale chronic disease epidemiology study. Its use has shown that MDD prevalence rates and risk factor profiles seem largely coincident with other work using more sophisticated assessment methods. In particular, little evidence was found for episode clustering just before respondent self assessment. The results reported were designed to expose any primary methodological weaknesses consequent upon the use of a modular self assessment approach to assessing psychiatric health status. As such we have yet to identify findings concerning putative HLEQ-MDD epidemiology that suggest divergence from those results already well established elsewhere, although there may be 
bias introduced through the limited compliance of eligible participants into EPIC. Such compliance was based upon participant commitment primarily to a longitudinal study examining diet diversity and cancer incidence, not a study of mental health epidemiology. The response rate obtained to the HLEQ from those already recruited to the study (about $77 \%$ ) is broadly coincident with that obtained in other large scale interviewer led studies but rather better that those dependent upon response to a mailed questionnaire.

We have been concerned here therefore with forging a bridgehead between the formal dogma of psychiatric epidemiology (the commitment to a binary approach to diagnosis), and the research opportunities that may arise through the study of very large cohorts where measures from multiple domains are available. In this process, it is probable that the basis for establishing the link to the traditional methods of psychiatric epidemiology, equivalence with MDD rates and risks, be abandoned in favour of measures of affective state (for example) that better reflect the inherent uncertainty attached to the diagnostic process. ${ }^{66}{ }^{67}$ Such indicators may eventually facilitate meeting the challenges of modelling the complex relations with the onset of disorder and with informing broader issues concerned with health service needs currently in debate..$^{68-70}$

We thank Professor Myrna Weissman, Division of Clinical and Genetic Epidemiology, Columbia University for commenting on an earlier draft of this paper and to Steven Greenwald, New York State Psychiatric Institute, for providing data output from the Epidemiologic Catchment Area study. The EPIC study in Norfolk is coordinated from Cambridge (UK). The project researchers in Cambridge are: Sheila Bingham, MRC Dunn researchers in Cambridge are: Sheila Bingham, MRC Dunn
Clinical Nutrition Centre; Kay-Tee Khaw, Department of Clinical Nutrition Centre; Kay-Tee Khaw, Department of and Ailsa Welch, Strangeways Research Laboratories. The project researchers associated with the psychosocial component of EPIC in Cambridge are: Carol Brayne, Department of Community Medicine and Institute of Public Health; Felicia Huppert, Department of Psychiatry; Chris Rolfe, Strangeways Research Laboratories; Paul Surtees and Nick Wainwright, MRC Biostatistics Unit and Institute of Public Health. The psychosocial component of EPIC in Norfolk is funded by the Medical Research Council. EPIC in Norfolk is supported by: British Heart Foundation, Cancer Research Campaign, Europe against Cancer Programme of the Commission of the European Communities, Imperial Cancer Research Fund, Medical Research Council, and the Ministry of Agriculture, Fisheries and Food.

Funding: the Medical Research Council.

Conflicts of interest: none.

1 Spitzer RL, Endicott J, Robins E. Research Diagnostic Criteria. Arch Gen Psychiatry 1978;35:773-82.

2 Robins LN, Helzer JE, Croughan J, et al. National Institute of Mental Health Diagnostic Interview Schedule: Its History, Characteristics and Validity. Arch Gen Psychiatry 1981;38:381-9.

3 Robins LN, Wing J, Wittchen H-U, et al. The Composite International Robins LN, Wing J, Wittchen H-U, et al. The Composite International
Diagnostic Interview: an epidemiological instrument suitable for use Diagnostic Interview: an epidemiological instrument suitable for use
in conjunction with different diagnostic systems and in different culin conjunction with different diagnostic syste
tures. Arch Gen Psychiatry 1988;45:1069-77.

4 Wing JK, Babor T, Brugha T, et al. SCAN: Schedules for Clinical Assessment in Neuropsychiatry. Arch Gen Psychiatry 1990;47:58993.

5 World Health Organisation. The ICD-10 classification of mental and behavioural disorders. Diagnostic criteria for research. Geneva: World Health Organisation, 1993.

6 American Psychiatric Association, APA. Diagnostic and statistical manual of mental disorders. 4th ed. Washington, DC: American Psychiatric Association, 1994. 7 Cross-National Collaborative Group. The changing rate of major
depression - cross-national comparisons. $¥ A M A$ 1992;268:3098105.

8 Weissman MM, Bland RC, Canino GJ, et al. Cross-national epidemiology of major depression and bipolar disorder. $F A M A$ 1996;276: 293-9.

9 Anthony JC, Folstein M, Romanoski AJ, et al. Comparison of the lay Diagnostic Interview Schedule and a standardized psychiatric diagnosis: experience in Eastern Baltimore. Arch Gen Psychiatry 1985;42:667-75

10 Parker G. Are the lifetime prevalence estimates in the ECA study accurate? Psychol Med 1987;17:275-82.

11 Simon GE, VonKorff M. Re-evaluation of secular trends in depression rates. Am $\mathcal{F}$ Epidemiol 1992;135:1411-22.
12 Fombonne E. Increased rates of depression: update of epidemiological findings and analytical problems. Acta Psychiatr Scand 1994;90:14556.

13 Simon GE, VonKorff M, Bedirhan Ustun T, et al. Is the lifetime risk of depression actually increasing? F Clin Epidemiol 1995;48:1109-18. 4 Helzer JE, Robins LN, McEvoy LT, et al. A comparison of clinical and diagnostic interview schedule diagnoses: physician reexamination of
lay-interviewed cases in the general population, Arch Gen Psychiatry

$1985 ; 42: 657-66$.
15 Robins LN. Epidemiology - reflections on testing the validity of psychiatric interviews. Arch Gen Psychiatry 1985;42:918-24.

16 Dohrenwend BP. The problem of validity in field studies of psychological disorders revisited. Psychol Med 1990;20:195-208,

Rice JP, Rochberg N, Endicott J, et al. Stability of psychiatric diagnoses 49:824-30.

18 Kendler KS, Neale MC, Kessler RC, et al. The lifetime history of major depression in women - reliability of diagnosis and heritability. Arch Gen Psychiatry 1993; 50:863-70.

19 Dohrenwend BP. A psychosocial perspective on the past and future of psychiatric epidemiology. Am f Epidemiol 1998;147:222-31.

20 Ader R, Cohen N, Felten D. Psychoneuroimmunology: interactions between the nervous system and the immune system. Lancet

21 Cohen S, Herbert TB. Health psychology: psychological factors and physical disease from the perspective of human psychoneuroimmuGoogy. A $\mathrm{K}$ A Rou Psy, Sevin B, at al. A par

Goodkin K, Antoni $\mathrm{MH}$, Sevin B, et al. A partially testable, predictive model of psychosocial factors in the etiology of cervical cancer. 2 immunological aspects, critique and prospective integration. Psychooncology 1993;2:99-122.

23 Anda R, Williamson D, Jones D, et al. Depressed affect, hopelessness, and the risk of ischemic heart disease in a cohort of U.S. adults. Epidemiology 1993;4:285-94.

24 Greenwood DC, Muir KR, Packham CJ, et al. Coronary heart disease: a review of the role of psychosocial stress and social support. $\mathcal{F}$ Public Health Med 1996;18:221-31.

25 Hippisley-Cox J, Fielding K, Pringle M. Depression as a risk factor for schaemic heart disease in men: population based case-control study.

Kawachi I, Colditz GA, Ascherio A, et al. A prospective study of social
networks in relation to total mortality and cardiovascular disease in men in the USA. F Epidemiol Community Health 1996;50:245-51.

27 Orth-Gomer K, Rosengren A, Wilhelmsen L. Lack of social support and incidence of coronary heart disease in middle-aged Swedish and incidence of coronary heart dised
men. Psychosom Med 1993;55:37-43.

28 Perrson L-G, Lindstrom K, Lingfors $\mathrm{H}$, et al. Interrelation between potential risk factors for cardiovascular disease in men aged $33-42$
years with special reference to life-style factors. Eur f Public Health years with special
$1994 ; 4: 238-44$

29 Nieto FJ. Cardiovascular disease and risk factor epidemiuology: a look back at the epidemic of the 20th century. Am $\mathcal{F}$ Public Health

30 Fox BH. The role of psychological factors in cancer incidence and prognosis. Oncology 1995;9:245-53. 31 Friedman GD. Invited commentary - depression, smoking, and
lung-cancer. Am F Epidemiol 1996;144:1104-6.

32 Linkins RW, Comstock GW. Depressed mood and development of cancer. Am f Epidemiol 1990;132:962-72.

33 Reynolds P, Kaplan GA. Social connections and risk for cancer: prospective evidence from the Alameda County Study. Behav Med 1990;16:101-10

34 Spiegel D, Kato PM. Psychosocial influences on cancer incidence and progression. Harv Rev Psychiatry 1996;4:10-26.

cer morbidity and mortality in a nationally representative for canfAMA 1989;262:1191-5.

36 Eaton WW, Armenian H, Gallo J, et al. Depression and risk for onset of Type-II diabetes. A prospective population-based study. Diabetes Care 1996;19:1097-102.

37 Everson SA, Goldberg DE, Kaplan GA, et al. Hopelessness and risk of mortality and incidence of myocardial infarction and cancer. Psychosom Med 1996;58:113-21.

38 Glassman AH, Shapiro PA. Depression and the course of coronary artery disease. Am f Psychiatry 1998;155:4-11.

Kaplan GA, Reynolds SP. Depression and cancer mortality and Behav Med 1988;11:1-13.

40 Kouzis A, Eaton WW, Leaf PJ. Psychopathology and mortality in the general-population. Soc Psychiatry Psychiatr Epidemiol 1995;30:16570

41 Morris PLP, Robinson RG, Andrzejewski P, et al. Association of depression with 10-year poststroke mortality. Am F Psychiatry 1993;

150:124-9.
42 Shekelle RB, Raynor WJ, Ostfeld AM, et al. Psychological depression and 17-year risk of death from cancer. Psychosom Med 1981;43:117

3 Pulska T, Pahkala K, Laippala P, et al. Follow up study of longstanding depression as predictor of mortality in elderly people living in the community. BMF 1999;318:432-33.

4 Penninx BWJH, Guralnik JM, Pahor $M$, et al. Chronically depressed 1998;90:1888-93.

45 Riboli E, Kaaks R. The EPIC Project: rationale and study design. Int $f$ Epidemiol 1997;26 (suppl 1):S6-14.

6 Bingham SA. Dietary assessments in the European prospective study of diet and cancer (EPIC). Eur F Cancer Prev 1997;6:118-24.

Bingham SA, Gill C, Welch A, et al. Validation of dietary assessment methods in the UK arm of EPIC using weighed records, and 24-hour urinary nitrogen and potassium and serum vitamin $\mathrm{C}$ and
carotenoids as biomarkers. Int $₹$ Epidemiol 1997;26 (suppl 1):S13751.

48 Day N, Oakes S, Luben R, et al. EPIC - Norfolk: study design and
characteristics of the cohort. Br f Cancer 1999;80(suppl 1):95-103. 49 Dillman DA. Mail and telephone surveys: the total design method. New York: Wiley, 1978

50 Ware JE. SF-36 health survey: manual and interpretation guide. Boston:

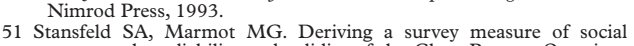
support: the reliability and validity of the Close Persons Question-

Kessler RC, McGonagle KA, Zhao S, et al. Lifetime and 12-month (he United States. Arch Gen Psychiatry 1994;51:8-19.

53 Bennett N, Dodd T, Flatley J, et al. Health survey for England 1993. London: HMSO, 1995.

54 Brazier JE, Harper R, Jones NMB, et al. Validating the SF-36 health survey questionnaire: new outcome measure for primary care. $B M \mathcal{F}$
1992;305:160-4. 
55 Jenkinson C, Coulter A, Wright L. Short Form-36 (SF-36) health survey questionnaire - normative data for adults of working age. $B M \mathcal{F}$ 1993;306:1437-40.

56 Lyons RA, Fielder H, Littlepage BNC. Measuring health status with the SF-36: the need for regional norms, I Public Health Med 1995;17 $46-50$

57 Jenkins R, Lewis G, Bebbington P, et al. The National Psychiatric Morbidity Surveys of Great Britain - initial findings from the Household Survey. Psychol Med 1997;27:775-89.

58 Weissman MM, Leaf PJ, Tischler GL, et al. Affective disorders in five United States communities. Psychol Med 1988;18:141-53.

59 Weissman $M M$, Olfson $M$. Depression in women: implications for health care research. Science 1995;269:799-801.

60 Bebbington P. The origins of sex differences in depressive disorder: bridging the gap. Int Rev Psychiatry 1996;8:295-332.

61 Bebbington P. Sex and depression. Psychol Med 1998;28:1-8.

62 Bebbington PE, Dunn G, Jenkins R, et al. The influence of age and sex on the prevalence of depressive conditions: report from the Nationa Survey of Psychiatric Morbidity. Psychol Med 1998;28:9-19.

63 World Health Organisation. World health report 1997: conquering suffer- ing, enriching humanity. Geneva: World Health Organisation, 1997

64 Fox BH. Premorbid psychological factors as related to cancer incidence. F Behav Med 1978;1:45-133.

65 Pratt LA, Ford DE Crum RM, et al. Depression, psychotropic medication, and the risk of myocardial infarction. Prospective data from cation, and the risk of myocardial infarction. Prospective data fro

Surtees PG. Wainwright NWJ, Gilks WR, et al. Diagnostic boundaries, reasoning and depressive disorder. II. Application of a probabilistic ity in Great Britain. Psychol Med 1997;27:847-60.

67 Wainwright NWJ, Surtees PG, Gilks WR. Diagnostic boundaries, reasoning and depressive disorder. I. Development of a probabilistic morbidity model for public health psychiatry. Psychol Med 1997;27: $835-45$.

68 Leeman E. Misuse of psychiatric epidemiology. Lancet 1998;351: $1601-2$

69 Regier DA, Kaelber CT, Rae DS, et al. Limitations of diagnostic criteria and assessment instruments for mental disorders. Implications for research and policy. Arch Gen Psychiatry 1998;55:109-15.

70 Spitzer RL. Diagnosis and need for treatment are not the same. Arch Gen Psychiatry 1998;55:20.

\section{Appendix}

\section{The next few questions are about your mood}

A1. Have there EVER been times in your life when you felt sad or depressed for TWO WEEKS or MORE in a row?

A2. Have there EVER been times in your life when you lost interest in most things like your work or activities that usually yes give you pleasure, for TWO WEEKS or MORE in a row?

$\begin{array}{ll}\text { yes } & \text { no } \\ \text { yes } & \text { no }\end{array}$

\section{IF NO to BOTH these} questions then please go to

\section{IF YES} next section.

For the next few questions, please think of the MOST RECENT TWO-WEEK episode during your life when these feelings of sadness, depression or loss of interest were worst.

A3. During that time did the feelings of being sad or depressed, or loss of interest usually last all day long, most of the day, about half the day or less than this? (please circle) All day long Most of the day About half the day Less than half the day

A4. During those two weeks, did you feel this way every day, almost every day or less often?
Every day
Almost every day
Less often

$\begin{array}{cc}1 & 2 \\ \text { During those two or more weeks did you:- }\end{array}$

A5. During those two or more weeks

a. $\quad$ gain or lose weight without trying?
b. $\quad$ have trouble falling asleep than you usually do, or sleeping too much?

c. feel tired out or low on energy all the time?

d. feel unable to sit still and had to keep moving or the opposite - feeling slowed down and having trouble moving?

e. feel guilty or ashamed of yourself for something you did or thought?

f. feel inferior or even worthless?

g. lose confidence in yourself?

h. have trouble concentrating, thinking, or making decisions?

think a lot about death, either your own, someone else's, or suicide?

A6. People differ in how much their day to day activities are affected when they feel sad or depressed or lose interest in the things that they normally enjoy.

The next few questions are about how you were affected by these feelings and experiences during this same time that you have just described.

a. During those two weeks (or more), how much did these feelings and experiences interfere with your life (so much that it kept you from working, including such things as housework, children, and other activities or from seeing friends or relatives)? (Please circle)
Often
Sometimes
Rarely
Never

b. During this time did you tell a doctor about these feelings or take any medication?

(Please circle)

yes no

yes no

yes no

yes no

yes no

yes no

yes no

yes no no

During this time did you seek help from anyone else, like a minister, or a friend, or did anyone suggest that you seek help?

\begin{tabular}{cc}
\multicolumn{2}{c}{ (Please circle) } \\
yes & no \\
1 & 2 \\
yes & no \\
1 & 2
\end{tabular}

A7a. About when did this MOST RECENT TIME in your life start when you felt sad, depressed or had lost interest in things? (Please circle ONE month, if possible, and complete year)

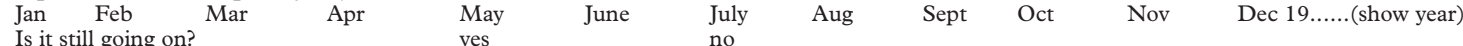
Is it still going on? $\quad$ yes $\quad$ no

IF NO

c. About when did this MOST RECENT TIME end? (Again, please circle ONE month, if possible, and complete year)

Jan Feb Mar Apr May June July Aug Sept Oct Nov Dec 19.....(show year) Can you remember your exact age the FIRST time that you experienced two weeks or more when you felt sad, depressed or lost interest in the things that you normally enjoyed?

(Please complete)

$1 \quad$ Yes, I can remember, I was .........................years of age.

2 No, I cannot remember exactly, but the earliest age that I can clearly remember having an episode like this and lasting for two weeks or more was when I was about .......................years of age.

A9. In your lifetime, about how many of these episodes have you had that lasted for two weeks or more when you felt sad, depressed or lost interest in things (and also had some of the other problems mentioned above)? (Please circle)

Number of episodes:

1 One

2 Two

4 Four

3 Three

5 At least ........... episode

6 Too many to remember

1 More than 2 weeks, but less than a month

2 One to six months

3 Six months to a year

4 Usually more than a year 